\title{
Height and dynamics of the quiet solar chromosphere at the limb
}

\author{
K. Muglach ${ }^{1}$ and W. Schmidt ${ }^{2}$ \\ 1 Astrophysikalisches Institut Potsdam, Telegrafenberg, 14473 Potsdam, Germany \\ 2 Kiepenheuer Institut für Sonnenphysik, Schöneckstraße 6, 79104 Freiburg, Germany
}

Received 8 June 2001 / Accepted 6 September 2001

\begin{abstract}
In this paper we present observations of $\mathrm{HeI} 1083.0 \mathrm{~nm}, \mathrm{HeD}_{3} 587.6 \mathrm{~nm}$ and $\mathrm{H} \beta 486.1 \mathrm{~nm}$ taken at various positions at the solar limb. We determine and compare the emission of the lines in terms of line-parameters. The height of the chromosphere as seen in the helium lines varies in space and time and reaches values between 1100 and $1800 \mathrm{~km}$ above the continuum and is the same for both helium lines within the errors of the measurement. From a time-sequence of slit-spectra of about 23 min we study the oscillation signature of the chromosphere near the solar limb. We find velocity oscillations in HeI $1083.0 \mathrm{~nm}$ that do not drop to zero near the limb as would be expected of vertically oriented oscillations, we even get horizontal oscillations in the off-limb emission data of both helium lines.
\end{abstract}

Key words. Sun: chromosphere - Sun: oscillations

\section{Introduction}

The spectral lines of neutral helium have been widely used to study the chromosphere of the sun and other stars. From ground, two such lines can be observed, $\mathrm{He}_{3} 587.6 \mathrm{~nm}$ in the optical and HeI $1083.0 \mathrm{~nm}$ in the near infrared (He IR). These lines can be used to map chromospheric activity, see e.g. synoptic solar images from Kitt Peak or Mauna Loa. In most cases they are optically thin (e.g. Giovanelli \& Hall 1977), unlike other chromospheric lines like $\mathrm{CaIIK}$ or $\mathrm{H} \alpha$. Thus, they have been used to study chromospheric dynamics like oscillations (e.g. Fleck et al. 1994; Bocchialini \& Baudin 1995; Baudin et al. 1996; Lites 1986) and high velocity events (e.g. Schmidt et al. 2000; Muglach et al. 1997; Andretta et al. 1999; Muglach \& Sütterlin 1999; Penn \& Allen 1997).

In addition, He I $1083 \mathrm{~nm}$ is the only tool to map coronal holes from ground which can easily be recognized on full disk He I $1083 \mathrm{~nm}$ images. The relationship of He IR and coronal radiation was studied in detail e.g. by Golub et al. (1989) who searched for a correlation of dark He IR absorption features and X-ray bright points and find a good correspondence. Thompson et al. (1993) also confirm a close relationship between chromospheric and coronal emission features and He IR absorption. This dual relationship hints towards a line formation mechanism that includes both the chromosphere and the corona. Coronal radiation of wavelengths shortward of the photoionization

Send offprint requests to: K. Muglach,

e-mail: kmuglach@aip.de threshold for the He ground state (at $50.4 \mathrm{~nm}$ ) shines onto the chromosphere and photoionizes helium atoms. These ions then recombine to the excited levels and produce He I lines. This is called the photoionisation-recombination (PR) process. Alternatively, collisional excitations can take place in regions with electron temperatures above $20000 \mathrm{~K}$, the lowest transition region. A detailed description of the two mechanisms and their relative importance for various features of the solar helium spectrum as well as extensive numerical calculations can be found in Andretta \& Jones (1997).

$\mathrm{D}_{3}$ and $\mathrm{He}$ IR are also sensitive to the magnetic field and especially recent Zeeman polarimetry gave first results on the chromospheric magnetic field (Harvey \& Hall 1971; Rüedi et al. 1995; Rüedi et al. 1996; Penn \& Kuhn 1995).

Both the optical $\mathrm{He}_{3}$ line and the near-infrared He I $1083.0 \mathrm{~nm}$ line go into emission beyond the solar limb and have been used to measure off-limb phenomina like prominences (e.g. Lin et al. 1998) or spicules (Venkatakrishnan et al. 1992).

In this paper we present the first simultaneous limb observations of the two He I lines that are available from ground. Due to the wide separation in wavelength such a measurement is not easy. Andretta (1996) was the first to get simultaneous observations of the two lines using the Fourier Transform Spectrometer fed by the McMathPierce solar telescope at Kitt Peak. He compared the equivalent width of the two lines on the disk as a function of activity. 
Table 1. Observational parameters: The first column gives the number of the data set, Col. 2 gives the location of the slit and Col. 3 the lines which were simultaneously observed. Columns 4 and 5 give the exposure times of the respective line and the number of spectra that were obtained in a sequence. Column 6 gives the direction of the slit with respect to the solar limb.

\begin{tabular}{cllccc}
\hline number of sequence & location & spectral lines & exposure times $[\mathrm{s}]$ & \# of spectra & comment \\
\hline$\# 1$ & east limb & He I 1083.0, He D & 10,2 & 38 & slit $\perp$ limb \\
$\# 2$ & east limb & He I 1083.0, He D & 10,3 & 94 & slit $\perp$ limb \\
$\# 3$ & west limb & He I 1083.0, He D & 10,3 & 12 & slit $\perp$ limb \\
$\# 4$ & north limb & He I 1083.0, H $\beta$ & 10,5 & 39 & slit $\|$ limb \\
\hline
\end{tabular}

Schmidt et al. (1994) carried out first measurements of the limb emission of He IR with the VTT on Tenerife. The current paper can be considered as an extension of their work. In particular, we will compare the two helium lines in terms of their observed line parameters like their off-limb distribution and their response to the dynamics in the solar chromosphere.

After this introduction we present the observations and details of the data reduction, followed by a description of the results of this analysis in Sect. 3. Finally, in Sect. 4, we conclude with a discussion of the results and compare them with previous measurements.

\section{Observations and reduction}

Our observations were carried out on 10th and 11th July, 1995, using the Echelle spectrograph of the German Vacuum Tower Telescope (VTT) on Tenerife, Spain. Two CCD cameras were used to record either HeI $1083.0 \mathrm{~nm}$ and $\mathrm{He}_{3} 587.6 \mathrm{~nm}$ or He I $1083.0 \mathrm{~nm}$ and $\mathrm{H} \beta 486.1 \mathrm{~nm}$ simultaneously.

The spatial resolution given by the pixel size of the CCD was $0.183^{\prime \prime}$ per pixel, the spatial resolution given by seeing was about $1-2^{\prime \prime}$. The spectral sampling was 1.35 , 0.72 and $0.59 \mathrm{pm}$ per pixel for the two helium lines and the hydrogen line, respectively.

The slit of the spectrograph (having a width of $100 \mu \mathrm{m}$, corresponding to $0.46^{\prime \prime}$ ) covered $94^{\prime \prime}$ and was located at various positions at the solar disk and the limb. Table 1 summarizes the four data sets we chose for the following analysis. As noted in Col. 6 of Table 1 the slit was positioned perpendicular to the solar limb and short time sequences were recorded in the first three data sets. In the last data set (\#4) the slit was parallel to the limb and we scanned over the limb with a stepsize of $0.4^{\prime \prime}$ (thus covering an area of about $\left.15^{\prime \prime} \times 94^{\prime \prime}\right)$. The time difference between the spectra was $15 \mathrm{~s}$.

We used the correlation tracker of the VTT (Schmidt \& Kentischer 1995) to stabilize the image to about $0.1 \operatorname{arcsec}(\mathrm{rms})$. Since the tracker sensor would not work at the limb, it was positioned on a small facular point on the disk, as close as possible to the spectrograph slit. Since the seeing was rather good, the tracking error due to offset pointing was negligible.

First, all CCD frames were corrected for dark and gain. In data set \#4 our observing procedure was different from the three others but the reduction procedure was carried out analogous.

Then we determine the continuum along the spatial direction by averaging an appropriate number of spectral pixels in the spectral continuum. This average continuum profile (continuum intensity vs. $x$ ) is subtracted from each spectrum to remove straylight, that is due to scattered light by seeing. Figure 1 shows a typical example of the resulting $\mathrm{HeD}_{3}$ (left) and $\mathrm{He} \mathrm{I} 1083.0 \mathrm{~nm}$ (right) spectra, in both cases it is the 10 th frame of set \#1: wavelength $\lambda$ is on the $x$-axis, spatial direction on the sun is on the $y$-axis, the gray shading gives the (linear) intensity. The figures show an area of $28^{\prime \prime}$ around the solar limb. In the lower part of the $\mathrm{He}_{3}$ image very weak telluric $\mathrm{H}_{2} \mathrm{O}$ lines can be seen, one of them almost exactly at the position of the helium line $(587.56 \mathrm{~nm})$ obscuring the signature of a possible He I absorption on the disk. Seeing conditions were mostly good and the water content of the air above Tenerife was very low, so we see practically no influence of the telluric line on the $\mathrm{D}_{3}$ emission (no aureole). On the right of Fig. 1 the same part of the simultaneously observed infrared helium line is shown. We see weak helium absorption on the disk and in the prominent emission beyond the limb the second component of the He I triplet at $1082.9 \mathrm{~nm}$ can well be recognized. The strong line at $1083.2 \mathrm{~nm}$ is again a terrestrial $\mathrm{H}_{2} \mathrm{O}$ line. Giovanelli \& Hall (1977) give quiet sun sample spectra as well as lineidentifications of this wavelength range.

Next, we determined the position of the solar limb using the same continuum data as mentioned in the text above. Two different methods were applied: (i) we fit the limb with a third order polynomial and determine the point of inflexion analytically (see Fig. 1 of Schmidt et al. 1994); (ii) the zero-crossing of the second derivative was calculated by linear interpolation of the adjacent points (this method was applied by Penn \& Jones 1996). The rms of the difference in the limb-position found by these two methods is $20-30 \mathrm{~km}$. Figure 2 gives the time variation of the limb-position for the first three data sets for both spectral lines, He I 1083.0 is represented by the solid lines, $\mathrm{He}_{3}$ by the dashed lines. The position of the limb of the first He I 1083.0 spectrum is arbitrarily set to 0 , all variations of the limb-position are converted to a distance (a difference of one pixel on the CCD equals to $133 \mathrm{~km}$ ).

Short term variations of the limb-position are due to seeing, so for this figure we applied a 3 -point smoothing 

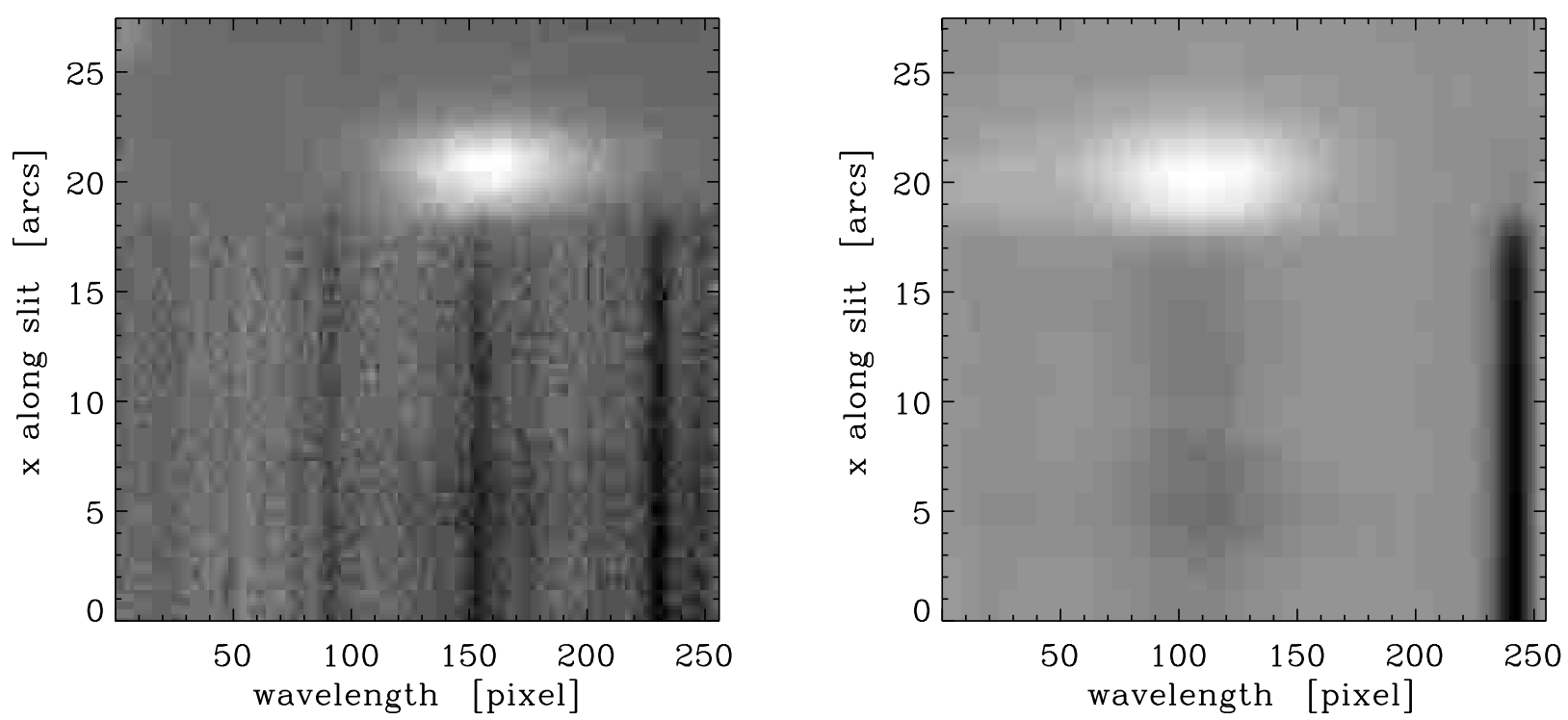

Fig. 1. Example of an emission spectrum of $\mathrm{He}_{3}$ (left) and He I 1083 (right) after removal of scattered light due to seeing.

in time. The time variation we see in Fig. 2 is the same for both spectral regions. The first half of set \#2 shows an oscillatory change of the limb-position with a 300 s period. The shift of the limb-position corresponds to a radial velocity of about $2 \mathrm{~km} \mathrm{~s}^{-1}$, which is in reasonable agreement with the properties of the global solar oscillations.

The fact that the limb-position of the two spectral regions is not the same is due to (i) a small offset of the two CCD cameras in the focal plane of the spectrograph and (ii) differential refraction of the Earths atmosphere. Another possibility would be a difference in the formation height of the continuum in the visible and the IR. Schmidt et al. (1994) as well as Penn \& Jones (1996) assume that both continua originate from the same height in the solar atmosphere. Any difference in their formation heights should be visible as a phase difference in the variation of the two limbs seen in Fig. 2, especially in set \#2. Figure 2 does not show any phase difference between the two limbs and we also get a phase $\phi=0^{\circ}$ for the $5 \mathrm{~min}$ period when actually calculating the phase spectrum of set \#2 (although the time sequence is quite short). Longterm image drifts (e.g. \#1) may be due to proper motion of the facular point we used for tracking or by a change in the facular points shape resulting in a slight motion of the lock point.

Finally, three line parameters of both helium lines were computed: the line-center wavelength $\lambda_{\text {cent }}$ (by fitting a parabola to the lowest part of the line), the line-center intensity $I_{\text {cent }}$ and the spectrally integrated intensity $I_{\text {int }}$ $( \pm 0.05 \mathrm{~nm}$ around the line minimum).

\section{Results}

\section{1. $\mathrm{He} \mathrm{D}_{3}$ and $\mathrm{He}$ I 1083.0 emission above the limb}

As can be seen in Fig. 1 the He I lines go into emission beyond the limb, the spectrally integrated intensity $I_{\text {int }}$ reaches a maximum and then drops to zero again.
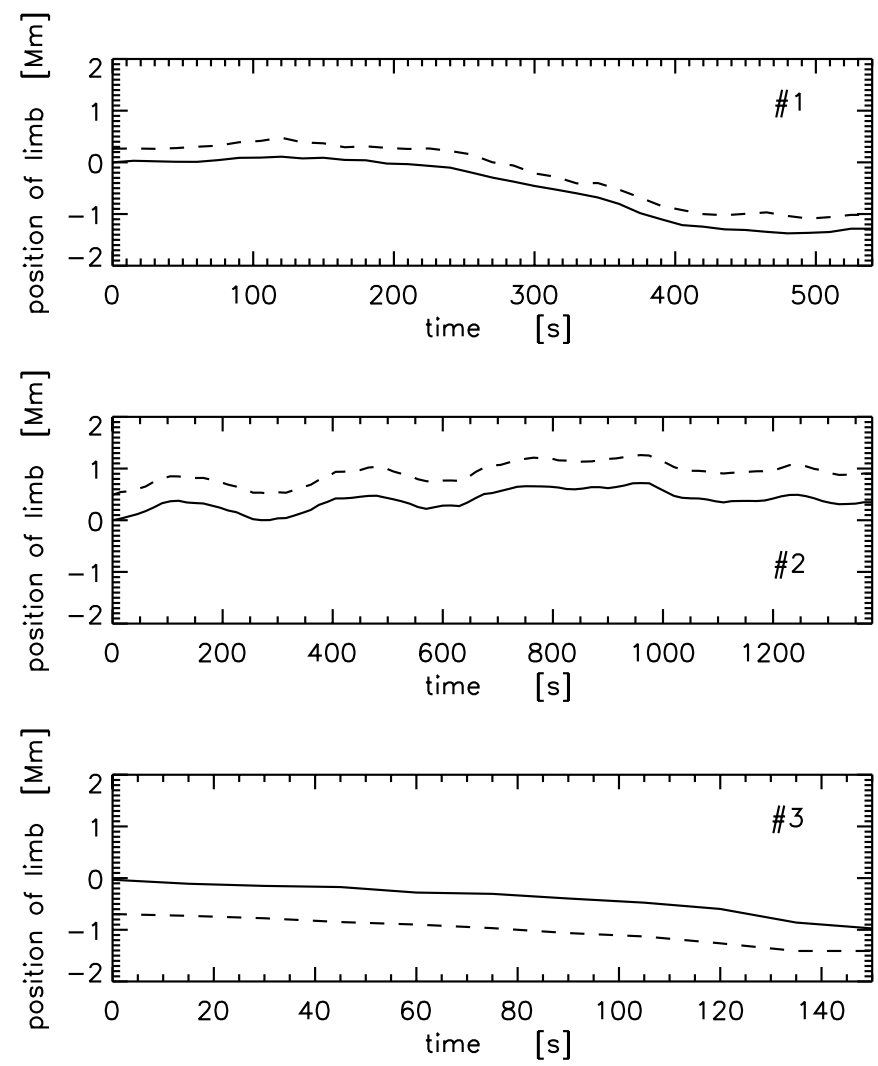

Fig. 2. Position of the solar limb on the CCD versus time of the first three data sets, $\mathrm{He}_{3}$ (dashed), HeI1083 (solid).

The location of the maximum emission was computed with a quadratic fit to the points around the emission peak. As the entrance slit of the spectrograph was perpendicular to the limb, the height of maximum helium emission is the difference between the position of the continuum limb and this location for each individual frame (thus taking into account the variation of the limb-position in Fig. 2). 

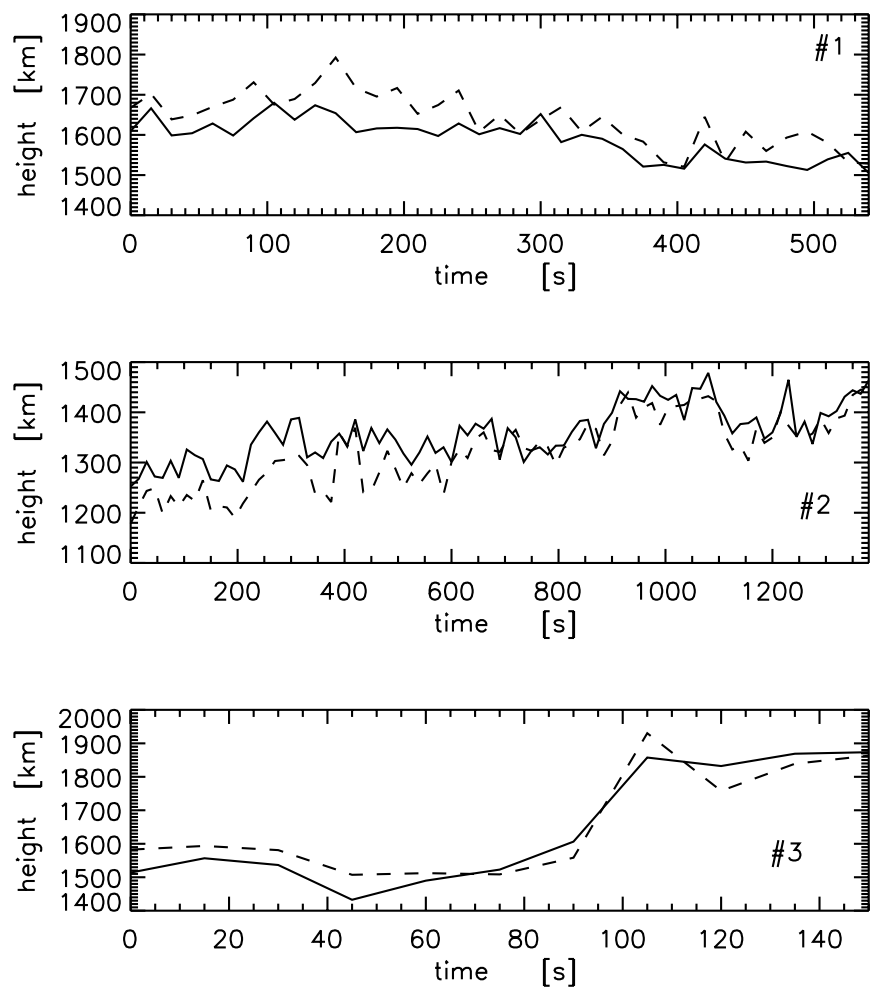

Fig. 3. Height of maximum of emission above the limb as shown in Fig. 2, $h_{\max }$, versus time of the first three data sets, $\mathrm{He}_{3}$ (dashed), He I 1083 (solid).

Figure 3 shows the resulting height of maximum emission, $h_{\max }$, for the three sequences and Fig. 4 the value of $I_{\text {int }}$ at this height. The error in the fit of the emission peak is about $30 \mathrm{~km}$, so together with the uncertainty of the limb-position $(\sim 20 \mathrm{~km})$ the short-term variation in Fig. 3 is mostly due to these errors and the difference for the two wavelength regions is mostly less than that.

No absolute calibration of the two lines has been carried out, so in Fig. 4 we added a constant value to the $\mathrm{D}_{3}$ emission to shift it to a comparable scale. Two things are apparent in Fig. 4: $I_{\text {int }}$ is remarkably constant over time (rms values are only a few percent) and the short-term variations are similar for the two wavelength regions.

\subsection{Limb-scan of $\mathrm{He} / 1083 \mathrm{~nm}$}

Sequence \#4 was taken at the north limb and the slit was positioned parallel to the limb. We scanned from the diskside towards the limb with 39 steps, $0.4^{\prime \prime}$ each, so we cover about $15^{\prime \prime} \times 94^{\prime \prime}$ with the spatial resolution of $0.8^{\prime \prime}$ perpendicular to the slit. In this case we observed He I $1083 \mathrm{~nm}$ and $\mathrm{H} \beta$. Again, we determine the limb from the continuum data and remove the continuum in the helium data. As $\mathrm{H} \beta$ is much broader than the wavelength range of our CCD, we cover only the central part of the line. Thus, we have no continuum to remove and we cannot apply the same analysis procedure as for the helium lines. In addition, as $\mathrm{H} \beta$ is optically thick and shows a much more complex transition of absorption on disk to emission
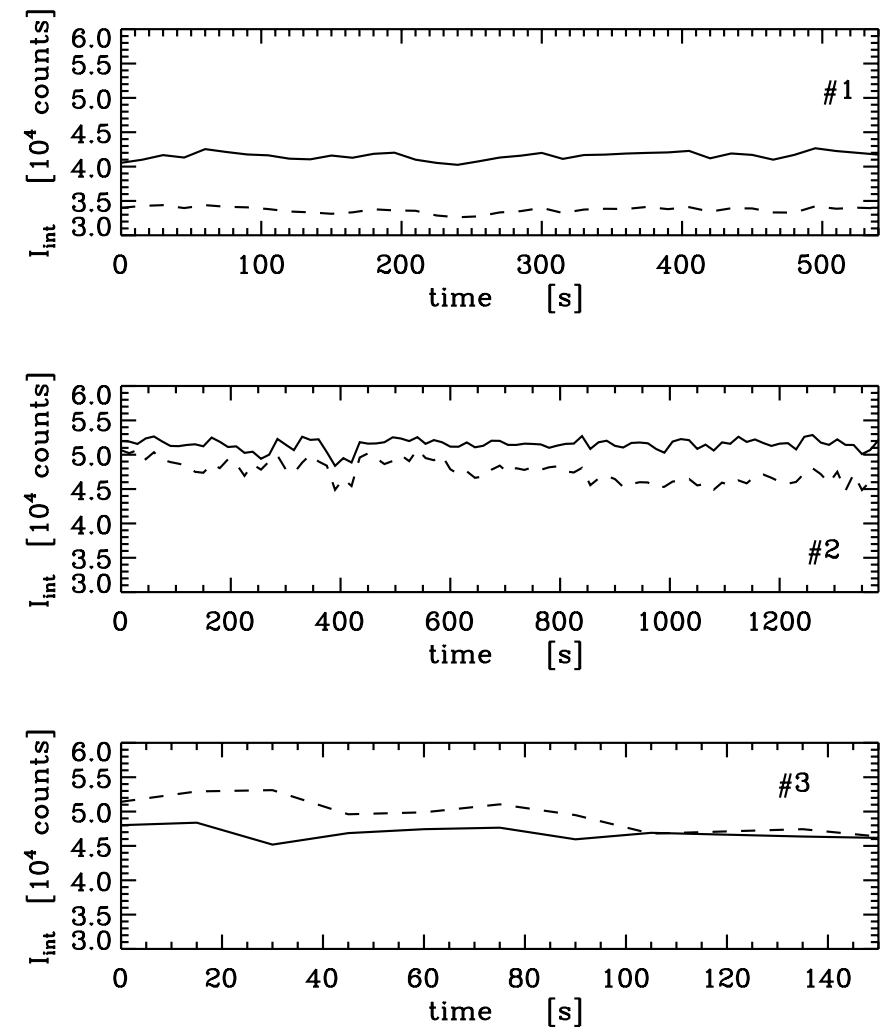

Fig. 4. Emission $I_{\text {int }}$ at $h_{\max }$ above the limb versus time of the first three data sets, He $\mathrm{D}_{3}$ (dashed), He I 1083 (solid).

off-limb, we refrain from a direct comparison of He IR and $\mathrm{H} \beta$.

For He IR we calculate the same line parameters as mentioned in the previous paragraph. Figure 5 shows the integrated helium emission $I_{\text {int }}$ along the limb. Finally, Fig. 6 shows $h_{\max }$ (top) and $I_{\text {int }}$ (bottom) at $h_{\max }$ along the northern limb of the solar disk (the slight tilt angle of radial direction has not been taken into account when calculating $\left.h_{\max }\right)$.

\subsection{Time sequence of He I lines, power spectra}

In this section we investigate the time variation of $\lambda_{\text {cent }}$ and $I_{\text {cent }}$ of both He I lines using data set \#2, which has a length of 23.5 min. The top of Fig. 7 shows line position $\lambda_{\text {cent }}$ of $\mathrm{He} \mathrm{D}_{3}$ (left) and He I 1083.0 (right) in a gray-scale image, on the $x$-axis is the spatial dimension along the slit (in "), on the $y$-axis is the time (in s). The IR image clearly shows the variation of Doppler shift of the line in time. Considering the fact that we are near the limb of the solar disk, these Doppler shifts correspond to more or less horizontal velocities. Although the visible line is almost completely obscured by the terrestrial blend on the disk (see Fig. 1), the image is not evenly gray. Some structure is nevertheless recognizable which is comparable to the one seen in the IR image although much weaker. The variation of the limb-position (seen in Fig. 2) is taken into account, thus the vertical structure around $x \approx$ $57^{\prime \prime}$ is the position where absorption goes into emission 


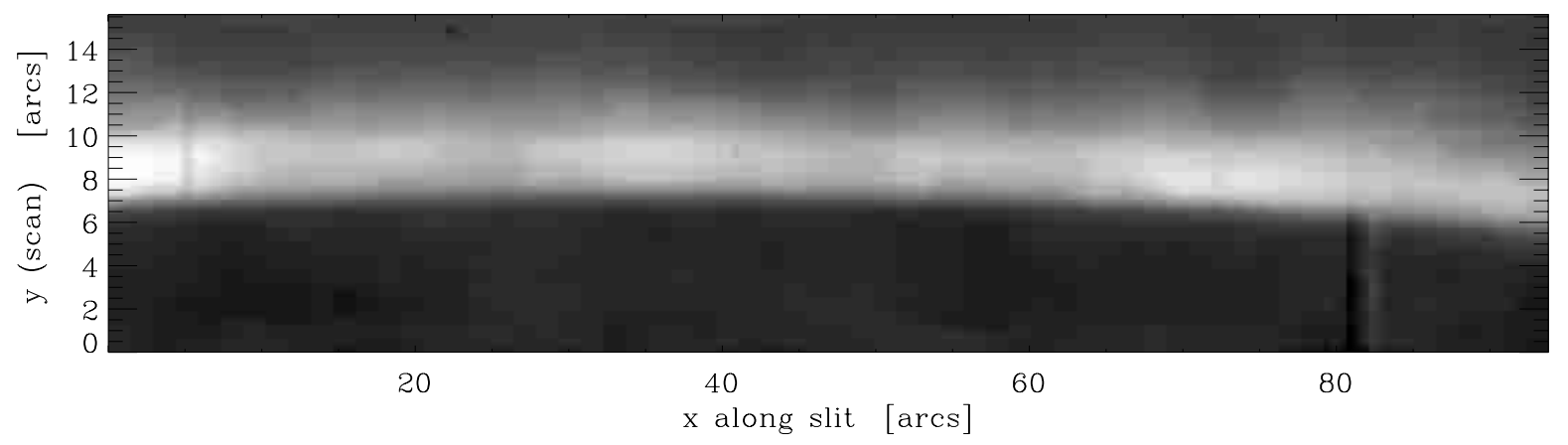

Fig. 5. $I_{\text {int }}$ of the north limb scan.

(the line profiles are too weak to give a reliable fit). Beyond $x \approx 57^{\prime \prime}$ lie the emission profiles of both lines. At $x=6^{\prime \prime}$ a hairline covered the entrance of the spectrograph slit and thus, there is no valid information.

The positions of $\mu=0.3,0.2$ and 0.1 are at $x=$ $13.4^{\prime \prime}, 38.2^{\prime \prime}$ and $52.8^{\prime \prime}$, respectively.

We calculated a power spectrum for each time sequence along the slit and show them in the second panel of Fig. 7. We inverted the colortable, so the dark patches represent places of enhanced power. We have chosen to show the power spectra up to $21 \mathrm{mHz}$, beyond this we get about the same amount of noise as between 15 and $21 \mathrm{mHz}$.

On disk the $\mathrm{He} \mathrm{D}_{3}$ power spectra do not show any signal but in the emission region we can clearly see some power. The IR data shows power for the disk spectra as well as for the off-limb spectra.

In the lower half of Fig. 7 we show the same for intensity instead of line position. Because of the strong emission beyond the limb we treated disk and off-limb data differently for this figure: on disk we show $I_{\text {cent }}$ as determined from the fit of the profiles, for the off-limb spectra we calculated a mean limb profile (averaged over time) and subtracted it. Thus the variation in the shadings seen in Fig. 7 (3rd panel) between $x=57^{\prime \prime}-67^{\prime \prime}$ shows the deviation to this mean profile. This procedure was not applied when calculating the intensity power spectra given in the lowest panel of Fig. 7. He $\mathrm{D}_{3}$ does not show anything on the disk again, off-limb emission shows some intensity fluctuations. The IR line shows some changes in intensity but in the power spectra we mostly see very low frequency signal.

There are two interesting frequency ranges, one around $3.3 \mathrm{mHz}(5 \mathrm{~min})$ and one around $5.5 \mathrm{mHz}(3 \mathrm{~min})$. Figure 8 gives the integrated power of these two frequency ranges along the slit (top: $4.2-6.4 \mathrm{mHz}$, bottom: $2.2-3.5 \mathrm{mHz}$ ) for the IR helium line, each for $\lambda_{\text {cent }}$ and $I_{\text {cent }}$. We note here, that we do not see a decrease in the power from the centerward end of the slit to the limb, as one would expect from a mainly radial wave field. See Sect. 4 for a more detailed discussion.

Finally, Fig. 9 gives an example of an off-limb time sequence (top) and power spectrum (bottom) in $\lambda_{\text {cent }}$ (left)
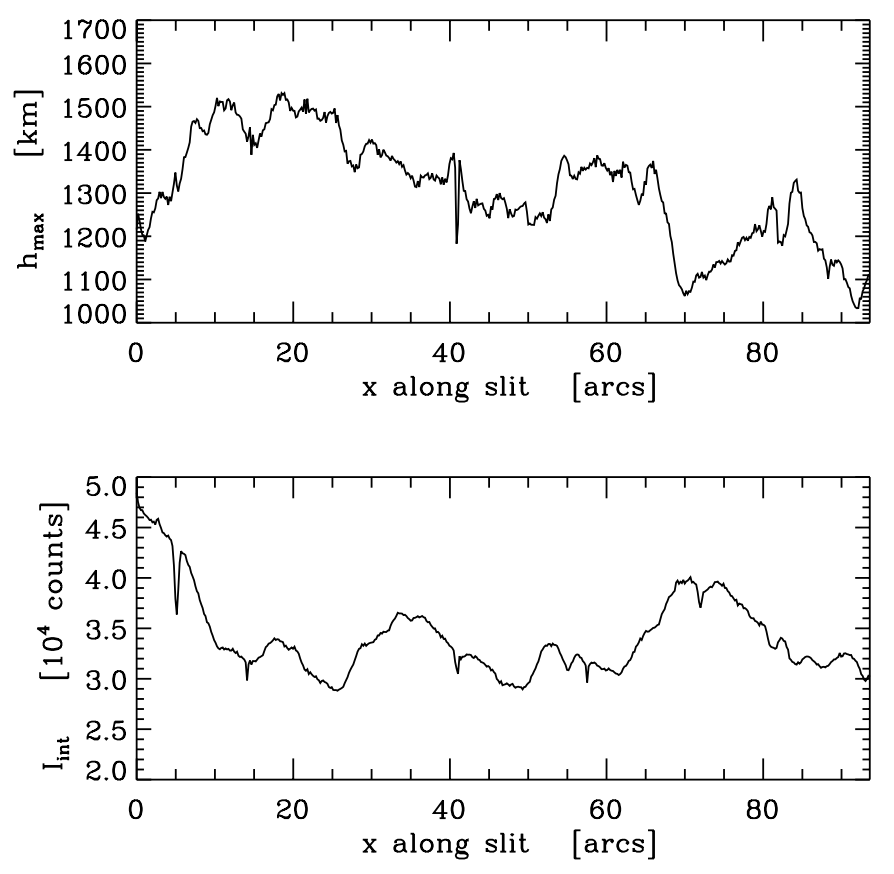

Fig. 6. $h_{\max }$ (top) and $I_{\text {int }}$ (bottom) at $h_{\max }$ along the north limb.

and $I_{\text {cent }}$ (right), at $x=60.4^{\prime \prime}$, spatially averaged over 5 pixel $\left(1^{\prime \prime}\right)$.

\section{Discussion}

As these are the first simultaneous high resolution spectroscopic observations of the two helium lines at the limb of the solar disk, our discussion emphasizes on a comparison of these two lines in terms of the observed line parameters.

The similarity in the behaviour of the continuum limb in the visible and the near IR (Fig. 2) implying a similar formation region of the two continua has already been mentioned in Sect. 2.

One of the central questions of this investigation concerns the height in the chromosphere where the two spectral lines originate. Figure 3 allows a direct comparison of them because they were observed strictly simultaneously, with the same instrument and the reduction and analysis procedure were the same as well. The difference in height that we find is within the errors of our measurement 

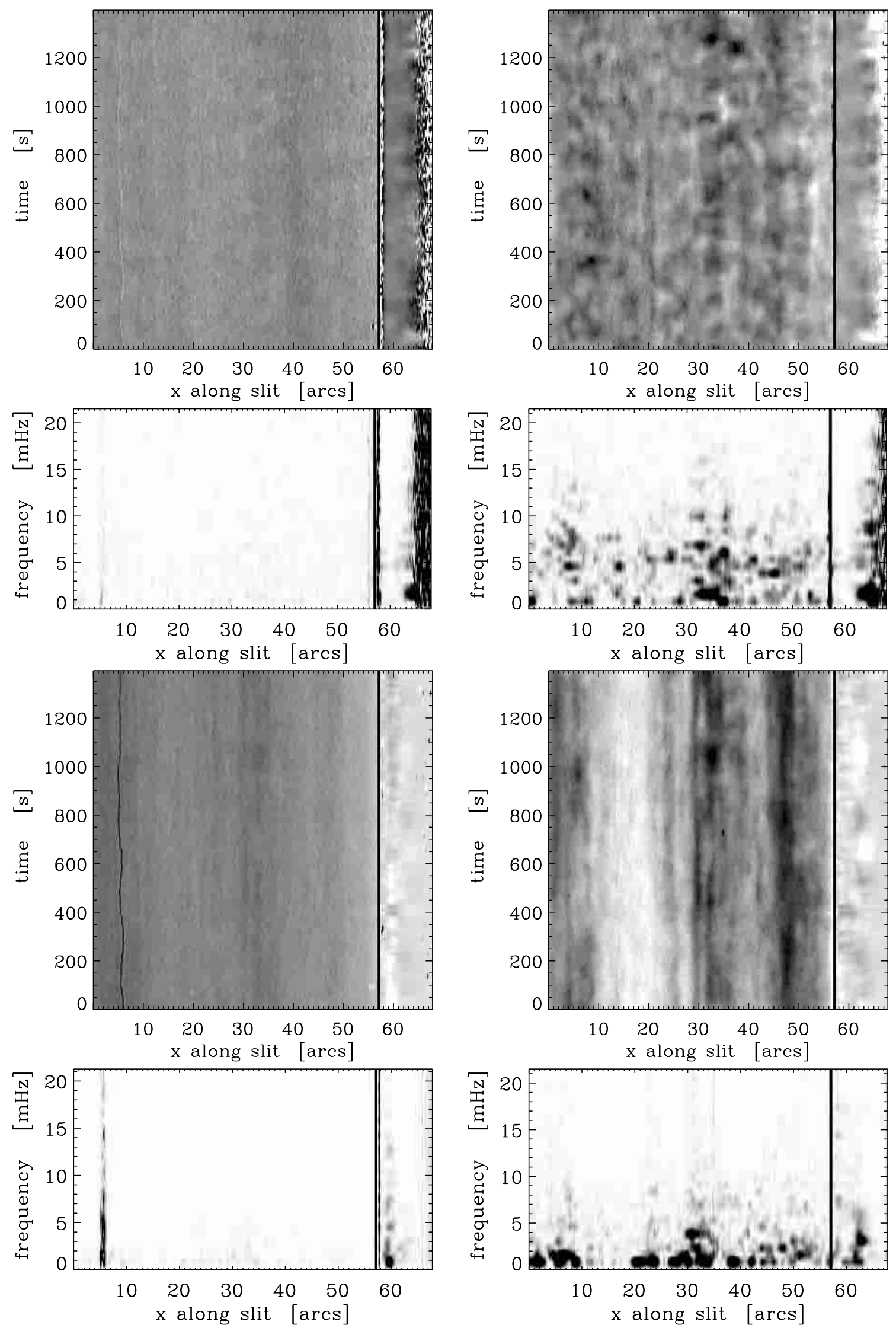

Fig. 7. Top panel: time variation of the line position $\lambda_{\text {cent }}$ of $\mathrm{He}_{3}$ (left) and He I 1083 (right) along the slit, at $x=57^{\prime \prime}$ corresponds to the solar limb, changes of the limb-position (see Fig. 2) have been removed; second panel: power spectra of $\lambda_{\text {cent }}$ along the slit; third panel: time variation of the line-center intensity $I_{\text {cent }}$ of $\mathrm{He}_{3}$ (left) and He I 1083 (right) along the slit; bottom panel: power spectra of $I_{\text {cent }}$ along the slit. All for data set \#2. 

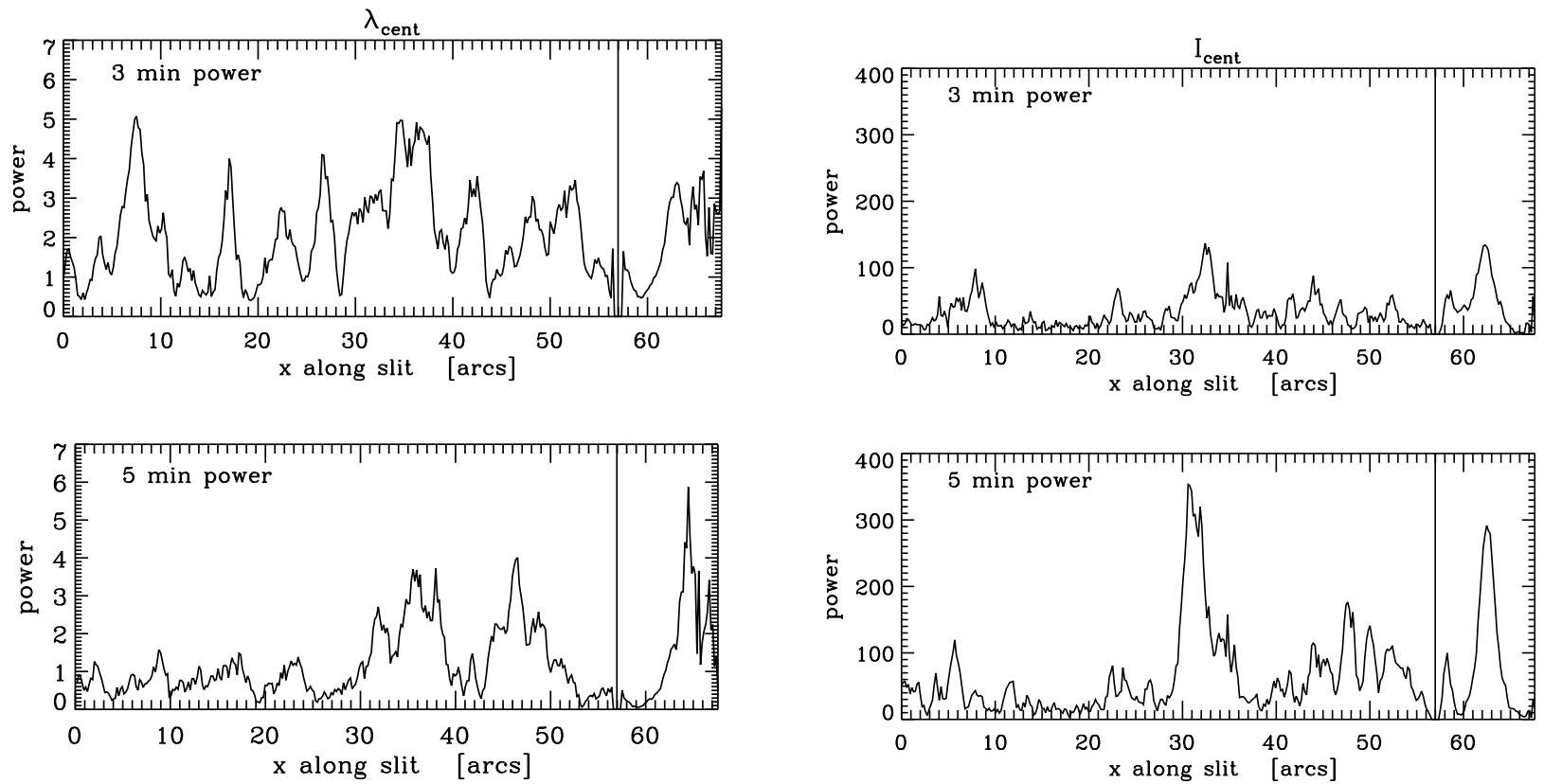

Fig. 8. Velocity (left) and intensity power (right) of He I $1083.0 \mathrm{~nm}$ along the slit in 2 frequency ranges (top: $4.2-6.4 \mathrm{mHz}$, bottom: $2.2-3.5 \mathrm{mHz}$ ), limb position is indicated at $x=57^{\prime \prime}$.
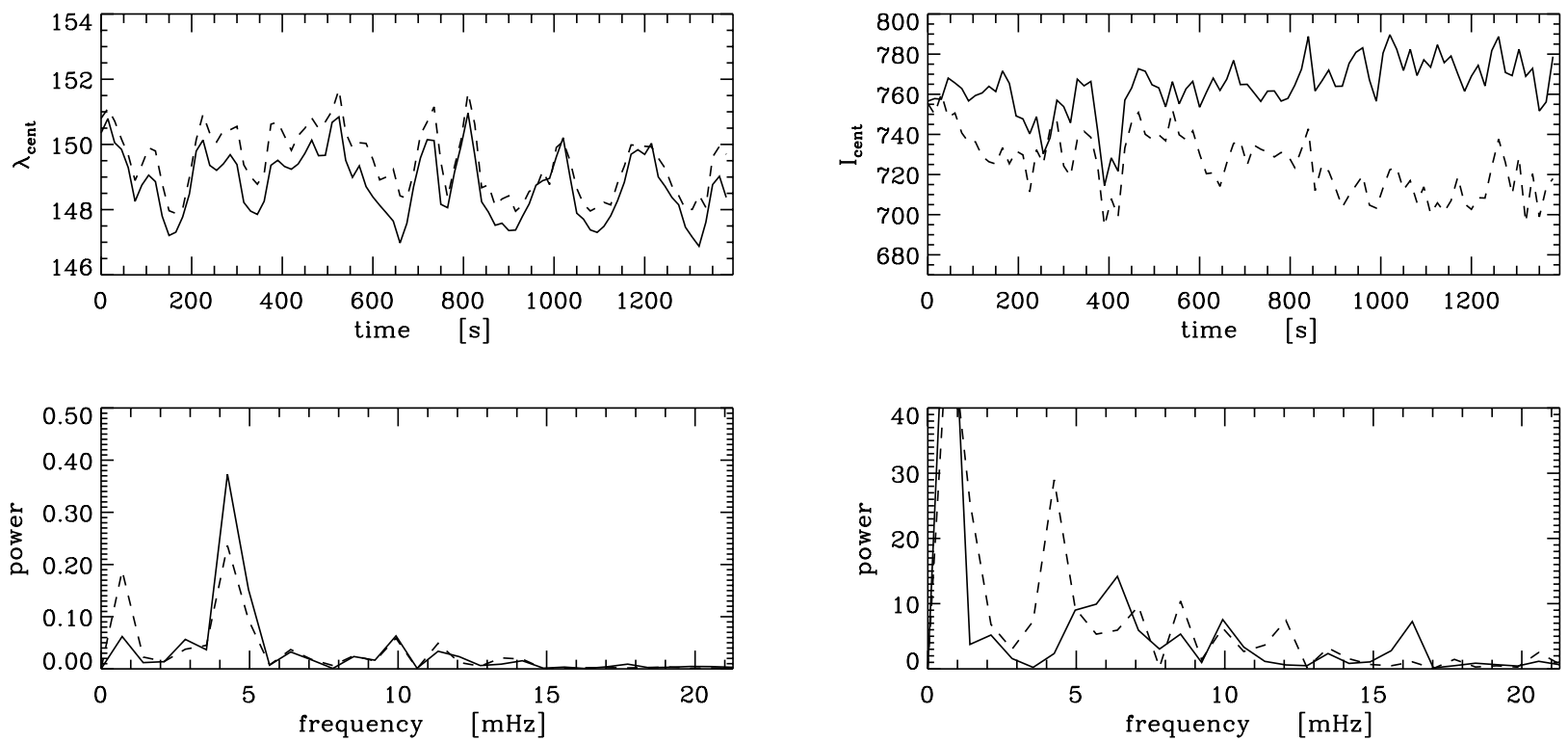

Fig. 9. Example of a single line plot (top) and power spectrum (bottom) at $x=60.4^{\prime \prime}$ (averaged spatially over 5 pixel, $1^{\prime \prime}$ ), on the left is velocity, on the right intensity, He $\mathrm{D}_{3}$ (dashed), He I 1083 (solid).

and analysis, which supports the idea that $\mathrm{He} \mathrm{D}_{3}$ and He I $1083.0 \mathrm{~nm}$ come from the same height in the quiet solar atmosphere and react very similarly to the atmospheric conditions there (changes in $I_{\text {int }}$ at $h_{\text {max }}$ of Fig. 4 are also very similar, we only have a constant offset in intensity between the two lines). We find considerable temporal as well as spatial variation of $h_{\max }$ : the time sequences of Fig. 3 range from rather constant values of 1600-1700 km (over $10 \mathrm{~min})$ in $\# 1$, to a slow increase from 1200 to $1500 \mathrm{~km}$ in about half an hour in \#2, and a sharp rise from 1500 to $1800 \mathrm{~km}$ within $1 \mathrm{~min}$ in $\# 3$. Including the results of data set \#4 in Fig. 6, taken at the north pole of the sun, we get values as low as $1100 \mathrm{~km}$ and up to $1500 \mathrm{~km}$ over a range of $94^{\prime \prime}$. Images of the Soft X-ray Telescope (SXT) of Yohkoh taken on the same day show that there was a large coronal hole at the north pole and we are sure that \#4 was obtained inside this coronal hole.

The values in Fig. 6 are still well within the range of the other three data sets taken at the east and west limb.

In the following we compare our measurements with heights found in the literature: Table I of Penn \& Jones (1996) summarizes $\mathrm{D}_{3}$ emission maxima obtained by various authors over the last 35 years and gives values between 1100 and $1800 \mathrm{~km}$. Their own He I $1083.0 \mathrm{~nm}$ 
measurements (Table II) result in heights of about $2000 \mathrm{~km}$ and Schmidt et al. (1994) report 2200-2400 km. These values are higher than ours but taking into account our own temporal and spatial variation of $h_{\max }$, we consider them within the possible range of values.

Figures 7-9 summarize our analysis of the $23.5 \mathrm{~min}$ time sequence $(\# 2)$. We measure line shifts due to the Doppler effect, thus we obtain the line-of-sight velocity of the plasma. As we approach the limb, the line-of-sight becomes more and more parallel to the solar surface and our off-limb emission measurements correspond to the horizontal component of the motion of the emitting plasma. Figures 7 (top half) and 8 (left) clearly show velocity oscillations in the $4-6 \mathrm{mHz}$ regime both on disk and off-limb (see also Fig. 9). Since the He I lines are optically thin outside of prominences (Giovanelli \& Hall 1977), one would expect any horizontal motion to cancel, but our spatial and temporal resolution is not sufficient to completely average out all horizontal velocities. The velocity signal is also the same in the two spectral lines, as can be seen in the example given in Fig. 9: the solid lines represent He I $1083.0 \mathrm{~nm}$ and the dashed ones $\mathrm{He}_{3}$ in both the upper and lower part of the figure.

Intensity oscillations (Fig. 7 (lower half) and Fig. 8 right) show very little power, except in the lowest frequencies. As our sequence is only $23.5 \mathrm{~min}$ long, we refrain from speculating about 10 or 15 min oscillations. The changes in intensity of the two spectral lines is the only other parameter that is not same, apart from the absolute intensity.

Quiet sun chromospheric oscillations have been studied many times, mostly by means of photometry and spectroscopy in the Ca II H and K line, He I 1083.0 nm, the infrared CO lines (see reviews e.g. Rutten \& Uitenbroek 1991; Rutten 1999). But all of them refer to disk center observations, thus, the suggested interpretations of the chromospheric oscillations consider the radial velocity field only (e.g. simulations of Carlsson \& Stein 1995, which successfully reproduce internetwork Ca II K bright grains are purely one-dimensional).

Solanki et al. (1996) observed quiet sun chromospheric oscillations near the limb or off-limb in emission. They show power spectra of infrared CO lines very near the limb $(\mu \approx 0.07-0.09)$. As the CO lines are formed in the cool part of the chromosphere, i.e. deeper than the He I lines it is not surprising that their power spectra (Fig. 2) look quite different from ours. Penn \& Allen (1997) calculate the center-to-limb variation of the velocity oscillation seen in He I $1083.0 \mathrm{~nm}$ and the photospheric Si I $1082.8 \mathrm{~nm}$ line. They find that the integrated power (in the $p$-mode frequency band of $2-6 \mathrm{mHz}$ ) decreases towards the limb in both lines and reaches about zero at the limb (determined from an extrapolation of the decrease of the integrated power) and they find no oscillation in their off-limb sequences. This does not agree with our results (see Figs. $7-$ $9)$. We think that this is probably due to their strong spatial averaging $\left(1.14^{\prime \prime} \times 36^{\prime \prime}\right.$ on disk and $6^{\prime \prime} \times 36^{\prime \prime}$ off-limb $)$ possibly in addition to their limited spectral and temporal resolution (which they sacrificed to obtain a large spatial sampling). Nevertheless, a larger sample of high resolution time sequences is needed from the VTT (and from other observatories) to confirm our measurements .

Several groups have studied spectral line shifts near the solar limb especially in coronal holes (although without temporal resolution). E.g. Dupree et al. (1996) look for the center to limb variation of the He I 1083.0 line in a more statistical way (using a few thousand line profiles). In coronal holes they find a systematic blue-ward line asymmetry in line profiles of the internetwork which they interpret as a radial outflow of the fast solar wind (this is in contradiction to measurements of Hassler et al. 1999, who find blueshifts of the coronal Ne VIII line in the network).

Peter (1999) observes blueshifts in the optically thick He I $58.4 \mathrm{~nm}$ line in coronal holes as well. But he does not interpret them as an outflow of the solar wind. Instead, he suggests that optical depth and radiative transfer effects are playing an important role in explaining his observations.

So what may be the nature of our observed horizontal oscillation? Kink modes of small scale magnetic elements would be a candidate. These kind of waves are of great interest in terms of chromospheric and coronal heating as they are easily excited due to their lower cut-off frequency, they do not suffer from radiative damping and do not produce shocks in higher layers of the atmosphere. Thus, they are capable of channeling energy from the convection zone into the corona. The excitation of these waves may either be due to collision of the fluxtubes with granules (Choudhuri et al. 1993) or by turbulence of the convection (Huang et al. 1995). Recently, kink waves of magnetic elements in the photosphere have been studied numerically by various groups (Ploner \& Solanki 1997; Hasan \& Kalkofen 1999; Hasan et al. 2000; Steiner et al. 1995, 1996), but hardly any direct observational evidence has been presented up to now (Ulrich 1996). Again, in a statistical sense we can interpret Stokes $V$ zero-crossing rms velocities observed near the limb by Martínez Pillet et al. (1997) as transverse oscillations of photospheric fluxtubes, but without temporal resolution one can not exclude buffeting by granular convection as a source of their observed Doppler shifts.

Kalkofen (1997) suggests kink modes of small scale magnetic elements to explain the oscillations in chromospheric network bright points (observed at disk center). These kink modes would produce a signature of transverse waves at photospheric levels but due to mode coupling turn into longitudinal waves at the chromospheric level (and thus explain the heating in the network bright points). From our current observations we cannot tell, if they are taken in the network or the internetwork though the latter is more likely. Again, additional observations including imaging data may shed more light on this possibility.

Another explanation can be, that we see the extension of the global $p$-modes. Photospheric $p$-mode oscillations in the quiet sun are primarily vertical as shown by 
Stix \& Wöhl (1974) and Schmidt et al. (1999). Nevertheless, $p$-modes with a large angular degree $l\left(l \gtrsim 10^{3}\right)$ can reach the chromosphere due to a tunneleffect (Dzhalilov et al. 2000, see their Fig. 2). As calculated in Dzhalilov et al. these $p$-modes have some of the properties we observe here: (i) the relation of the horizontal to vertical velocity is large, so they are better seen near the limb, (ii) the modes are almost adiabatical, so they show very little intensity oscillation, (iii) the horizontal lengthscale is a few $1000 \mathrm{~km}$ (as seen in Fig. 8, left), (iv) $3 \mathrm{~min}$ periods can more easily tunnel though the barrier.

\section{Summary}

In this paper we have presented the first simultaneous measurements of $\mathrm{He}_{3}$ and $\mathrm{He}$ I $1083 \mathrm{~nm}$ at the limb of the quiet sun. We have compared the derived line parameters and found that the two lines give the same value of the chromospheric height (between 1100 and $1800 \mathrm{~km}$ above the continuum limb) and they show the same off-limb response to the dynamics of the chromospheric plasma. The near limb velocity oscillations that we find do not show a decrease in amplitude as would be expected of a radial wave-field. A possible mechanism to produce such horizonal waves would be kink modes of small scale magnetic elements. Or we might see the extension of high $-l$ acoustic $p$-modes that penetrate into the chromospheric layers of the sun.

Acknowledgements. The VTT is operated on the island Tenerife by the Kiepenheuer Institut für Sonnenphysik in the Spanish Observatorio del Teide of the Instituto de Astrofisica de Canarias. We would like to thank Louise Harra-Murnion for providing the Yohkoh SXT images. Yohkoh is a mission of the Institute of Space and Astronautical Science of Japan. This research was partially supported by the European Commission through the TMR programme (European Solar Magnetometry Network). We would like to thank H. Balthasar, H. Peter and J. Staude for comments on the manuscript.

\section{References}

Andretta, V. 1996, BAAS, 28(2)

Andretta, V, \& Jones, H. P. 1997, ApJ, 489, 375

Andretta, V., Jordan, S. D., Muglach, K., et al. 1999, ASP Conf. Ser., 155, 336

Avrett, E. H., Fontenla, J. M., \& Loeser, R. 1994, IAU Colloq. 154, Infra-red Solar Physics, 35, ed. D. M. Rabin, J. T. Jeffries, \& C. Lindsey

Baudin, F., Bocchialini, K., \& Koutchmy, S. 1996, A\&A, 314, L9

Bocchialini, K., \& Baudin, F. 1995, A\&A, 299, 893

Carlsson, M., \& Stein, R. F. 1997, ApJ, 481, 500
Choudhuri, A. R., Auffret, H., \& Priest, E. R. 1993, Sol. Phys., 143,49

Dzhalilov, N. S., Staude, J., \& Arlt, K. 2000, A\&A, 361, 1127

Dupree, A. K., Penn, M. J., \& Jones, H. P. 1996, ApJ, 467, L121

Fleck, B., Deubner, F.-L., \& Maier, D. 1994, IAU Colloq. 154, Infra-red Solar Physics, 65, ed. D. M. Rabin, J. T. Jeffries, \& C. Lindsey

Giovanelli, R. G., \& Hall, D. 1977, Sol. Phys., 52, 211

Golub, L., Harvey, K. L., Herant, M., \& Webb, D. F. 1989, Sol. Phys., 124, 211

Harvey, J. W., \& Hall, D. N. B. 1971, in Solar Magnetic Fields, IAU Symp., 43, 279

Hasan, S. S., \& Kalkofen, W. 1999, ApJ, 519, 899

Hasan, S. S., Kalkofen, W., \& van Ballegooijen, A. A. 2000, ApJ, 535, L67

Hassler, D. M., Dammasch, I. E., Lemaire, P., et al. 1999, Science, 283, 810

Huang, P., Musielak, Z. E., \& Ulmschneider, P. 1995, A\&A, 297, 579

Kalkofen, W. 1997, ApJ, 486, L145

Lites, B. 1986, ApJ, 301, 1005

Lin, H., Penn, M. J., \& Kuhn, J. R. 1998, ApJ, 493, 978

Martínez Pillet, V., Lites, B. W., \& Skumanich, A. 1997, ApJ, 474,810

Muglach, K., Schmidt, W., \& Knölker, M. 1997, Sol. Phys., 172,103

Muglach, K., \& Sütterlin, P. 1999, ASP Conf. Ser., 155, 341

Penn, M. J., \& Jones, H. P. 1996, Sol. Phys., 168, 19

Penn, M. J., \& Allen, C. L. 1997, Sol. Phys., 174, 359

Penn, M. J., \& Kuhn, J. R. 1995, ApJ, 441, L51

Peter, H. 1999, ApJ, 522, L77

Ploner, S. R. O., \& Solanki, S. K. 1997, A\&A, 325, 1199

Rutten, R. J. 1999, Third Advances in Solar Physics Euroconference: Magnetic Fields and Oscillations, ed. B., Schmieder, A., Hofmann, J., \& Staude, ASP Conf. Ser., 184, 181

Rutten, R.J., \& Uitenbroek, H. 1991, Sol. Phys., 134, 15

Rüedi, I., Solanki, S. K., \& Livingston, W. C. 1995, A\&A, 293, 252

Rüedi, I., Keller, C. U., \& Solanki, S. K. 1996, Sol. Phys., 164, 265

Schmidt, W., \& Kentischer, T. 1995, A\&AS, 113, 363

Schmidt, W., Knölker, M., \& Westendorp Plaza, C. 1994, A\&A, 287, 229

Schmidt, W., Stix, M., \& Wöhl, H. 1999, A\&A, 346, 633

Schmidt, W., Muglach, K., \& Knölker, M. 2000, ApJ, 544, 567

Solanki, S. K., Livingston, W., Muglach, K., \& Wallace, L. 1996, A\&A, 315, 303

Steiner, O., Grossmann-Doerth, U., Knölker, M., \& Schüssler, M. 1995, Rev. Mod. Astron., 8, 81

Steiner, O., Grossmann-Doerth, U., Knölker, M., \& Schüssler, M. 1996, Sol. Phys., 164, 223

Stix, M., \& Wöhl, H. 1974, Sol. Phys., 37, 63

Thompson, W. T., Neupert, W. M., Jordan, S. D., et al. 1993, Sol. Phys., 147, 29

Venkatakrishnan, P., Jain, S. K., Singh, J., Recely, F., \& Livingston, W. C. 1992, Sol. Phys., 138, 107 